\title{
The Fractional Quadratic-Form Identity and Hamiltonian Structure of an Integrable Coupling of the Fractional Broer-Kaup Hierarchy
}

\author{
Chao Yue, ${ }^{1,2}$ Tiecheng Xia, ${ }^{2}$ Guijuan Liu, ${ }^{1}$ and Jianbo Liu ${ }^{3}$ \\ ${ }^{1}$ College of Information Engineering, Taishan Medical University, Taian 271016, China \\ ${ }^{2}$ Department of Mathematics, Shanghai University, Shanghai 200444, China \\ ${ }^{3}$ Scientific Research Department, Taishan Medical University, Taian 271016, China \\ Correspondence should be addressed to Guijuan Liu; liugjok@163.com
}

Received 21 May 2013; Revised 4 August 2013; Accepted 26 August 2013

Academic Editor: Bo Yu

Copyright (c) 2013 Chao Yue et al. This is an open access article distributed under the Creative Commons Attribution License, which permits unrestricted use, distribution, and reproduction in any medium, provided the original work is properly cited.

\begin{abstract}
A fractional quadratic-form identity is derived from a general isospectral problem of fractional order, which is devoted to constructing the Hamiltonian structure of an integrable coupling of the fractional BK hierarchy. The method can be generalized to other fractional integrable couplings.
\end{abstract}

\section{Introduction}

The theory of derivatives of noninteger order can go back to Leibniz, Liouville, Grunwald, Letnikov, and Riemann. And the fractional analysis has attracted increasing interest of many researchers, because fractional analysis has numerous applications: kinetic theories $[1-3]$, such as statistical mechanics [4-6], dynamics in complex media $[7,8]$, and many others [9-16]. In recent studies in physics, the researchers have found many applications of the derivatives and integrals of fractional order $[16,17]$. They also pointed out that fractional-order models are more appropriate than integer-order models for various real materials. The main advantage of fractional derivative in comparison with classical integer-order models is that it provides an effective instrument for the description of memory and hereditary properties of various materials and progress. Also, the advantages of the fractional derivatives become apparent in modeling mechanical and electrical properties of real materials and in the description of rheological properties of rocks, as well as in many other fields.

The fractional calculus is a generalization of ordinary differentiation and integration to arbitrary order [17-20]. Since Riewe $[4,21]$ presented a concept of nonconservation mechanics, fractional conservation laws [22], Lie symmetries [9], and fractional Hamiltonian systems [23-33] have been receiving more and more attention.

It is an important and interesting topic to search for new Hamiltonian hierarchies of soliton equations and their integrable couplings in soliton theory. Tu once proposed a simple and efficient method to construct the integrable systems and Hamiltonian structures [34], which was called the Tu scheme by Ma [35]. Later, many integrable systems and their Hamiltonian structures were worked out [36-39]. Recently, $\mathrm{Wu}$ and Zhang proposed the generalized Tu formula and searched for the Hamiltonian structure of fractional AKNS hierarchy [40]. In [41], a generalized Hamiltonian structure of the fractional soliton equation hierarchy was presented. Very recently, Wang and Xia obtained the fractional supersoliton hierarchies and their super-Hamiltonian structures by using fractional supertrace identity $[42,43]$. Then, how to generate integrable coupling system and Hamiltonian structure of fractional soliton equation?

In this paper, begining with a general isospectral problem of fractional order, we propose a fractional quadraticform identity, from which the Hamiltonian structure of an integrable coupling of the fractional BK hierarchy is derived. 


\section{Brief Overview of Fractional Differentiable Functions}

Several local versions have been presented [44-52], among which Jumarie's derivative is defined as follows [52]:

$$
\begin{array}{r}
D_{x}^{\alpha} f(x)=\frac{1}{\Gamma(1-\alpha)} \frac{d}{d x} \int_{0}^{x}(x-\xi)^{-\alpha}(f(\xi)-f(0)) d \xi, \\
(0<\alpha<1) ;
\end{array}
$$

some properties of the fractional differentiable functions are given as follows.

(a) The Leibniz product law.

Assuming that $f(x)$ is an $\alpha$ order differentiable function in the area of point $x$, from the Jumarie-Kolwankar's Taylor series [52-54], we can have

$$
D_{x}^{\alpha} f(x)=\lim _{y \rightarrow x^{+}} \frac{\Gamma(1+\alpha)(f(y)-f(x))}{(y-x)^{\alpha}}, \quad(0<\alpha \leq 1) .
$$

If $g(x)$ is a differentiable function of $\alpha$ order, the Leibniz product law can hold for the nondifferentiable functions [39, $44,45]$

$$
D_{x}^{\alpha}(f(x) g(x))=g(x) D_{x}^{\alpha} f(x)+f(x) D_{x}^{\alpha} g(x) .
$$

(b) Denoting ${ }_{0} I_{x}^{\alpha}$ as the Riemann-Liouville integration in the following form:

$$
\begin{array}{r}
{ }_{0} I_{x}^{\alpha} f(x)=D_{x}^{-\alpha} f(x)=\frac{1}{\Gamma(\alpha+1)} \int_{0}^{x} f(\xi)(d \xi)^{\alpha}, \\
(0<\alpha \leq 1),
\end{array}
$$

we can have a generalized Newton-Leibniz formulation

$$
\begin{gathered}
\frac{1}{\Gamma(1+\alpha)} \int_{0}^{1} D_{x}^{\alpha} f(x)(d x)^{\alpha}=f(1)-f(0), \\
\frac{1}{\Gamma(1+\alpha)} \int_{0}^{x} D_{x}^{\xi} f(\xi)(d \xi)^{\alpha}=f(x)-f(0), \\
\frac{D_{x}^{\alpha}}{\Gamma(1+\alpha)} \int_{0}^{x} f(\xi)(d \xi)^{\alpha}=f(x) .
\end{gathered}
$$

(c) With the properties (a) and (b), integration by parts for $\alpha$ order differentiable functions $f(x)$ and $g(x)$ can be generated as

$$
\begin{aligned}
& \frac{1}{\Gamma(1+\alpha)} \int_{a}^{b} g(x) D_{x}^{\alpha} f(x)(d x)^{\alpha} \\
& \quad=\left.g(x) f(x)\right|_{a} ^{b}-\frac{1}{\Gamma(1+\alpha)} \int_{a}^{b} f(x) D_{x}^{\alpha} g(x)(d x)^{\alpha} .
\end{aligned}
$$

(d) From $[31,32,55]$, the fractional variational derivative is written as

$$
\frac{\delta L}{\delta y}=\frac{\partial L}{\partial y}+\sum_{k=1}(-1)^{k}\left(D_{x}^{\alpha}\right)^{k}\left(\frac{\partial L}{\partial\left(D_{x}^{\alpha}\right)^{k} y}\right)
$$

where $k$ is a positive integer. In this paper, we propose a generalized quadratic-form identity for fractional soliton hierarchy from (7).

\section{Fractional Exterior Differential and Hamiltonian Equations}

Since Adda proposed the fractional generalization of differential forms [56, 57], several versions of fractional exterior differential approaches and applications related to different forms of fractional derivatives appeared in some parts of the open literature $[58,59]$. The properties of fractional derivatives are discussed in [60].

The exterior derivative is defined as

$$
d=\sum_{m=1}^{n} d x_{m} \frac{\partial}{\partial x_{m}}
$$

The exterior derivative map $k$ forms into $k+1$ forms and has the following algebraic results. Let $\gamma$ and $\lambda$ be $k$ forms, and let $\mu$ be an $m$ form; we have

$$
\begin{gathered}
d(\gamma+\lambda)=d \gamma+d \lambda, \\
d(\gamma \wedge \mu)=(d \gamma) \wedge \mu+(-1)^{k} \gamma \wedge d \mu, \\
d(d \gamma)=0 .
\end{gathered}
$$

The last identity is called the Poincaré lemma. A form $\gamma$ is called closed if $d \gamma=0$. A form $\gamma$ is called exact if there exists a form $\mu$ such that $d \mu=\gamma$. The order of $\mu$ is one less than the order of $\gamma$. Exact forms are always closed, closed forms are not always exact.

Next, we introduce the fractional exterior derivative

$$
d^{\alpha}=\left(d x_{i}\right)^{\alpha} D_{x_{i}}^{\alpha}
$$

A differential 1-form is defined by

$$
\omega_{\alpha}=F^{i}(x)\left(d x_{i}\right)^{\alpha}
$$

with the vector field $F^{i}(x)$ that can be represented as $F^{i}(x)=-D_{x_{i}}^{\alpha} V$ and $V(x)$ is a continuously differentiable function. Using (10), the exact fractional form can be expressed as

$$
\omega_{\alpha}=-d^{\alpha} V=-\left(d x_{i}\right)^{\alpha} D_{x_{i}}^{\alpha} V
$$

Note that (11) is a fractional generalization of the differential form (8). It is easy to find that fractional 1-form $\omega_{\alpha}$ can be closed when the differential 1-form $\omega=\omega_{1}$ is not closed.

Then, we define the fractional functional

$$
J[p, q]=\frac{1}{\Gamma(1+\alpha)} \int\left[p D_{t}^{\alpha} q-H(t, p, q)\right](d t)^{\alpha}
$$

hence, we can readily derive the generalized Poincare-Cartan 1 -form, which reads

$$
\omega=p d^{\alpha} q-H(d t)^{\alpha}
$$


From (14), one has

$$
\begin{aligned}
d^{\alpha} \omega= & p_{t}^{\alpha}(d t)^{\alpha} \wedge d^{\alpha} q+d^{\alpha} p \wedge d^{\alpha} q \\
& -\frac{\partial H}{\partial p} d^{\alpha} p \wedge(d t)^{\alpha}-\frac{\partial H}{\partial q} d^{\alpha} q \wedge(d t)^{\alpha} \\
= & {\left[p_{t}^{\alpha}+\frac{\partial H}{\partial q}\right](d t)^{\alpha} \wedge d^{\alpha} q } \\
& +\left[\frac{\partial H}{\partial p}(d t)^{\alpha}-d^{\alpha} q\right] \wedge d^{\alpha} p .
\end{aligned}
$$

In the previous derivation, $p$ and $q$ are fractional differentiable functions with respect to $t$.

The fractional closed condition $d^{\alpha} \omega=0$ admits the fractional Hamilton's equations [40]

$$
q_{t}^{(\alpha)}=\frac{\partial H}{\partial p}, \quad p_{t}^{(\alpha)}=-\frac{\partial H}{\partial q},
$$

which can be generalized to the following case [31]:

$$
q_{i}^{(\alpha)}(t)=\frac{\partial H}{\partial p_{i}}, \quad p_{i}^{(\alpha)}(t)=-\frac{\partial H}{\partial q_{i}},
$$

\section{The Fractional Quadratic-Form Identity}

Guo and Zhang once proposed quadratic-form identity [61], which is very efficient tool to systematically generate integrable couplings and their Hamiltonian structures. In the following, the fractional quadratic-form identity is presented.

Set $G$ to be an $s$-dimensional Lie algebra with the basis

$$
e_{1}, e_{2}, \ldots, e_{s}
$$

whose corresponding loop algebra $\widetilde{G}$ possesses the following basis:

$$
\begin{gathered}
e_{i}(m)=e_{i} \lambda^{m}, \quad i=1,2, \ldots, s \\
m=0, \pm 1, \pm 2, \ldots, \\
{\left[e_{i}(m), e_{j}(m)\right]=\left[e_{i}, e_{j}\right] \lambda^{m+n} .}
\end{gathered}
$$

In terms of $\widetilde{G}$, we construct the following isospectral problem:

$$
\psi_{x}^{(\alpha)}=[U, \psi], \quad \psi_{t}^{(\beta)}=[V, \psi] .
$$

The compatibility condition of (20) gives rise to the generalized zero curvature equation:

$$
U_{t}^{(\beta)}-V_{x}^{(\alpha)}+[U, V]=0 .
$$

Taking $\alpha=\beta=1$ (21) reduces to the classical zero curvature equation. For $\lambda$ and $u_{i}(i=1,2, \ldots, p)$ in $U=U(\lambda, u)=$ $\sum_{i=1}^{s} U_{i} e_{i}$, defining $\operatorname{rank}(\lambda)=\operatorname{deg}(\lambda)$, then $\operatorname{rank}\left(e_{i}(\lambda)\right)=\alpha_{i}$, $0 \leq i \leq s$ can be presented. If the ranks of $u_{i}$ are taken as $\zeta-\alpha_{i}, 1 \leq i \leq s$, then each term in $U$ has the homogeneous rank $\alpha$ which is denoted by

$$
\operatorname{rank}(U)=\operatorname{rank}\left(\frac{\partial^{\alpha}}{\partial x^{\alpha}}\right)=\zeta
$$

Set $V=\sum_{m \geq 0} V_{m} \lambda^{-m}, V_{m}=\sum_{i=1}^{s} V_{m i} e_{i} \in G$, as a solution of the stationary zero curvature equation

$$
-V_{x}^{(\alpha)}+[U, V]=0
$$

and $\operatorname{rank}\left(V_{m}\right)_{\lambda}$ is assumed to be given so that $\operatorname{rank}\left(V_{m}\right)_{\lambda}=\xi$, $m \geq 0$; each team in $V$ has the same rank as follows:

$$
\operatorname{rank}(V)=\operatorname{rank}\left(\frac{\partial^{\beta}}{\partial t^{\beta}}\right)=\xi
$$

Let the two arbitrary solutions $V_{1}$ and $V_{2}$ of (23) with the same rank be linearly related by

$$
\bar{V}=\gamma V, \quad \gamma=\text { const. }
$$

In the following, relation (25) will be used when deducing the fractional quadratic-form identity. For $a, b \in \widetilde{G}$, the $s$-order matrix $R(b)$ is determined by

$$
[a, b]^{T}=a^{T} R(b)
$$

and constant matrix $F=\left(f_{i j}\right)_{s \times s}$ is determined by

$$
F=F^{T}, \quad R(b) F=-(R(b) F)^{T} .
$$

Defining functional $\{a, b\}=a^{T} F b$ satisfies the symmetry

$$
\{a, b\}=\{b, a\}
$$

and the bilinear relation

$$
\left\{c_{1} a_{1}+c_{2} a_{2}, b\right\}=c_{1}\left\{a_{1}, b\right\}+c_{2}\left\{a_{2}, b\right\} .
$$

In the sense of the local fractional derivative, the gradient $\nabla_{b}\{a, b\}$ of the functional $\{a, b\}$ is defined by

$$
\frac{\partial}{\partial \epsilon}\{a, b+\epsilon V\}=\left(\delta_{b}\{a, b\}, V\right), \quad a, b, V \in \widetilde{G}
$$

where $\delta_{b}$ is variational derivative with respect to $b$. With the fractional variational derivative (7), one can have

$$
\delta_{b}\left\{a, b_{x}^{(k \alpha)}\right\}=(-1)^{k} a_{x}^{(k \alpha)}
$$

where $k$ is a positive integer and $D_{x}^{k \alpha}=\underbrace{D_{x}^{\alpha} \cdots D_{x}^{\alpha}}_{k}$. The communication relationship of $\{a, b\}$ can be given as

$$
\{[a, b], c\}=\{a,[b, c]\}, \quad a, b, c \in \widetilde{G}
$$

Introduce a functional

$$
W=\left\{V, U_{\lambda}\right\}+\left\{\Lambda, V_{x}^{(\alpha)}-[U, V]\right\},
$$


where $U, V$ meet (23), while $\Lambda(\in \widetilde{G})$ is to be determined; using (7), we can obtain the following fractional variation constraint conditions:

$$
\frac{\delta W}{\delta \Lambda}=V_{x}^{(\alpha)}-[U, V], \quad \frac{\delta W}{\delta V}=U_{\lambda}-\Lambda_{x}^{(\alpha)}+[U, \Lambda]
$$

according to the Jacobi identity and the previous equations, we can have

$$
[\Lambda, V]_{x}^{\alpha}=\left[U_{\lambda}, V\right]+[U,[\Lambda, V]]
$$

$Z=[\Lambda, V]-V_{\lambda}$ and $V / \lambda$ are solutions of (23); using (25) and $\operatorname{rank}(Z)=\operatorname{rank}\left(V_{\lambda}\right)=\operatorname{rank}(V / \lambda)$, due to $V / \lambda$ satisfying (34), we can have $Z=(\gamma / \lambda) V$. From (23) and (33), a fractional quadratic-form identity is firstly presented as follows:

$$
\begin{aligned}
\frac{\delta}{\delta u_{i}}\left\{V, U_{\lambda}\right\} & =\left\{V, \frac{\partial U_{\lambda}}{\partial u_{i}}\right\}+\left\{[\Lambda, V], \frac{\partial U}{\partial u_{i}}\right\} \\
& =\left\{V, \frac{\partial U_{\lambda}}{\partial u_{i}}\right\}+\left\{V_{\lambda}, \frac{\partial U}{\partial u_{i}}\right\}+\frac{\gamma}{\lambda}\left\{V, \frac{\partial U}{\partial u_{i}}\right\} \\
& =\frac{\partial}{\partial \lambda}\left\{V, \frac{\partial U}{\partial u_{i}}\right\}+\left(\lambda^{-\gamma} \frac{\partial}{\partial \lambda} \lambda^{\gamma}\right)\left\{V, \frac{\partial U}{\partial u_{i}}\right\} \\
& =\lambda^{-\gamma} \frac{\partial}{\partial \lambda}\left(\lambda^{\gamma}\left\{V, \frac{\partial U}{\partial u_{i}}\right\}\right), \quad 1 \leq i \leq p .
\end{aligned}
$$

\section{Application of the Fractional}

Quadratic-Form Identity

Introduce a loop algebra $\widetilde{G}_{6}=\left\{a=\left(a_{1}, a_{2}, \ldots, a_{6}\right)^{T}, a_{k}=\right.$ $\left.\sum_{m} \lambda^{m}\right\}$, with the commuting relations

$$
\begin{aligned}
{[a, b]=(} & a_{2} b_{3}-a_{3} b_{2}, 2 a_{1} b_{2}-2 a_{2} b_{1}, 2 a_{3} b_{1} \\
& -2 a_{1} b_{3}, a_{2} b_{6}-a_{6} b_{2}+a_{5} b_{3}-a_{3} b_{5}, 2 a_{1} b_{5} \\
& -2 a_{5} b_{1}+2 a_{4} b_{2}-2 a_{2} b_{4}, 2 a_{6} b_{1} \\
& \left.-2 a_{1} b_{6}+2 a_{3} b_{4}-2 a_{4} b_{3}\right)^{T} .
\end{aligned}
$$

Consider the following spectral problem:

$$
\begin{gathered}
\psi_{x}^{(\alpha)}=[U, \psi], \\
U=\left(-\lambda+\frac{v}{2}, 1,-w, u_{1}, 0, u_{2}\right)^{T} \\
V=(a, b, c, d, e, f)^{T} .
\end{gathered}
$$

Solving equation

$$
-V_{x}^{(\alpha)}+[U, V]=0
$$

leads to

$$
\begin{gathered}
a_{m x}^{(\alpha)}=c_{m}+w b_{m}, \\
b_{m x}^{(\alpha)}=-2 b_{m+1}+v b_{m}-2 a_{m}, \\
c_{m x}^{(\alpha)}=2 c_{m+1}-v c_{m}-2 w a_{m}, \\
d_{m x}^{(\alpha)}=f_{m}+w e_{m}-u_{2} b_{m}, \\
e_{m x}^{(\alpha)}=-2 e_{m+1}+v e_{m}-2 d_{m}+2 u_{1} b_{m}, \\
f_{m x}^{(\alpha)}=2 f_{m+1}-v f_{m}-2 w d_{m}-2 u_{1} c_{m}+2 u_{2} a_{m}, \\
a_{1}=d_{1}=e_{1}=c_{2}=0, \quad b_{1}=1, \quad c_{1}=-w, \\
f_{1}=u_{2}, \quad a_{2}=-\frac{1}{2} w, \\
b_{2}=\frac{1}{2} v, \quad d_{2}=\frac{1}{2} u_{2}, \\
f_{2}=\frac{1}{2} u_{2 x}^{(\alpha)}+\frac{1}{2} u_{2} v-w u_{1}, \ldots
\end{gathered}
$$

Set

$$
\begin{gathered}
V^{(n)}=\sum_{m=0}^{n}\left(a_{m}, b_{m}, c_{m}, d_{m}, e_{m}, f_{m}\right)^{T} \lambda^{n-m} \\
+\left(b_{n+1}, 0,0,0,0,0\right)^{T} ;
\end{gathered}
$$

then the generalized zero curvature equation, $D_{t}^{\beta} U-D_{x}^{\alpha} V^{(n)}+$ $\left[U, V^{(n)}\right]=0$, gives rise to a system

$$
\begin{aligned}
& u_{t_{n}}^{(\beta)}=\left(\begin{array}{c}
v_{t_{n}}^{(\beta)} \\
w_{t_{n}}^{(\beta)} \\
u_{1 t_{n}}^{(\beta)} \\
u_{2 t_{n}}^{(\beta)}
\end{array}\right)=\left(\begin{array}{c}
2 b_{n+1, x}^{(\alpha)} \\
-2 a_{n+1, x}^{(\alpha)} \\
e_{n+1, x}^{(\alpha)} \\
2 d_{n+1, x}^{(\alpha)}
\end{array}\right) \\
& =\left(\begin{array}{cccc}
0 & 0 & 0 & 2 D_{x}^{\alpha} \\
0 & 0 & -D_{x}^{\alpha} & 0 \\
0 & -D_{x}^{\alpha} & 0 & -D_{x}^{\alpha} \\
2 D_{x}^{\alpha} & 0 & -D_{x}^{\alpha} & 0
\end{array}\right) \\
& \times\left(\begin{array}{c}
a_{n+1}+d_{n+1} \\
-b_{n+1}-e_{n+1} \\
2 a_{n+1} \\
b_{n+1}
\end{array}\right)=J P_{n+1},
\end{aligned}
$$

where $J$ is a Hamiltonian operator. From (40), we have a recurrence operator 


$$
L=\left(\begin{array}{cccc}
\frac{1}{2} D_{x}^{\alpha}+\frac{1}{2} D_{x}^{-\alpha} v D_{x}^{\alpha} & \frac{1}{2}\left(w+D_{x}^{-\alpha} w D_{x}^{\alpha}\right) & \frac{1}{2} D_{x}^{-\alpha} u_{1} D_{x}^{\alpha} & \frac{1}{2}\left(u_{2}+D_{x}^{-\alpha} u_{2} D_{x}^{\alpha}\right) \\
1 & \frac{1}{2}\left(v-D_{x}^{\alpha}\right) & 0 & -u_{1} \\
0 & 0 & \frac{1}{2}\left(D_{x}^{\alpha}+D_{x}^{-\alpha} v D_{x}^{\alpha}\right) & -w-D_{x}^{-\alpha} w D_{x}^{\alpha} \\
0 & 0 & -\frac{1}{2} & \frac{1}{2}\left(v-D_{x}^{\alpha}\right)
\end{array}\right)
$$

which meets $P_{n+1}=L P_{n}$. Hence, expression (42) can be written as

$$
u_{t_{n}}^{(\beta)}=\left(\begin{array}{c}
v_{t_{n}}^{(\beta)} \\
w_{t_{n}}^{(\beta)} \\
u_{1 t_{n}}^{(\beta)} \\
u_{2 t_{n}}^{(\beta)}
\end{array}\right)=J L^{n}\left(\begin{array}{c}
0 \\
-1 \\
0 \\
1
\end{array}\right) .
$$

From expression (37), we have

$$
\begin{gathered}
{[a, b]^{T}=a^{T}\left(\begin{array}{cccccc}
0 & 2 b_{2} & -2 b_{3} & 0 & 2 b_{5} & -2 b_{6} \\
b_{3} & -2 b_{1} & 0 & b_{6} & -2 b_{4} & 0 \\
-b_{2} & 0 & 2 b_{1} & -b_{5} & 0 & 2 b_{4} \\
0 & 0 & 0 & 0 & 2 b_{2} & -2 b_{3} \\
0 & 0 & 0 & b_{3} & -2 b_{1} & 0 \\
0 & 0 & 0 & -b_{2} & 0 & 2 b_{1}
\end{array}\right)} \\
=a^{T} R(b) .
\end{gathered}
$$

Solving the matrix equation (27) for $F$ leads to

$$
F=\left(\begin{array}{llllll}
2 & 0 & 0 & 2 & 0 & 0 \\
0 & 0 & 1 & 0 & 0 & 1 \\
0 & 1 & 0 & 0 & 1 & 0 \\
2 & 0 & 0 & 0 & 0 & 0 \\
0 & 0 & 1 & 0 & 0 & 0 \\
0 & 1 & 0 & 0 & 0 & 0
\end{array}\right)
$$

Let

$$
\begin{aligned}
\{a, b\}= & 2\left(a_{1}+a_{4}\right) b_{1}+\left(a_{3}+a_{6}\right) b_{2}+\left(a_{2}+a_{5}\right) b_{3} \\
& +2 a_{1} b_{4}+a_{3} b_{5}+a_{2} b_{6} ;
\end{aligned}
$$

we have

$$
\begin{gathered}
\left\{V, \frac{\partial U}{\partial v}\right\}=a+d, \quad\left\{V, \frac{\partial U}{\partial w}\right\}=-b-e, \\
\left\{V, \frac{\partial U}{\partial u_{1}}\right\}=2 a, \quad\left\{V, \frac{\partial U}{\partial u_{2}}\right\}=b, \\
\left\{V, \frac{\partial U}{\partial \lambda}\right\}=-2 a-2 d .
\end{gathered}
$$

Substituting the previous results into the fractional quadraticform identity (36) gives

$$
\frac{\delta}{\delta u}(-2 a-2 d)=\lambda^{-\gamma} \frac{\partial}{\partial \lambda} \lambda^{\gamma}\left(\begin{array}{c}
a+d \\
-b-e \\
2 a \\
b
\end{array}\right)
$$

Comparing the coefficients of $\lambda^{-n-1}$ on both sides of (49) yields

$$
\frac{\delta}{\delta u}\left(-2 a_{n+1}-2 d_{n+1}\right)=(\gamma-n)\left(\begin{array}{c}
a_{n}+d_{n} \\
-b_{n}-e_{n} \\
2 a_{n} \\
b_{n}
\end{array}\right)
$$

It is easy to find that $\gamma=0$; then we obtain the fractional Hamiltonian structure of (42)

$$
u_{t_{n}}^{(\beta)}=\left(\begin{array}{c}
v_{t_{n}}^{(\beta)} \\
w_{t_{n}}^{(\beta)} \\
u_{1 t_{n}}^{(\beta)} \\
u_{2 t_{n}}^{(\beta)}
\end{array}\right)=J\left(\begin{array}{c}
a_{n+1}+d_{n+1} \\
-b_{n+1}-e_{n+1} \\
2 a_{n+1} \\
b_{n+1}
\end{array}\right)=J \frac{\delta H_{n}}{\delta u}
$$


where $H_{n}=\left(2 a_{n+1}+2 d_{n+1}\right) / n$ and $(n=0,1,2, \ldots)$ is the fractional Hamiltonian function. When taking $n=2$, we have an integrable coupling of a fractional BK hierarchy

$$
\begin{gathered}
D_{t_{2}}^{\beta} v=-\frac{1}{2} D_{x}^{\alpha} D_{x}^{\alpha} v+v D_{x}^{\alpha} v+D_{x}^{\alpha} w \\
D_{t_{2}}^{\beta} w=\frac{1}{2} D_{x}^{\alpha} D_{x}^{\alpha} w+D_{x}^{\alpha}(w v) \\
D_{t_{2}}^{\beta} u_{1}=-\frac{1}{2} D_{x}^{\alpha} D_{x}^{\alpha} u_{1}+D_{x}^{\alpha}\left(u_{1} v\right)-\frac{1}{2} D_{x}^{\alpha} u_{2} \\
D_{t_{2}}^{\beta} u_{2}=\frac{1}{2} D_{x}^{\alpha} D_{x}^{\alpha} u_{2}+D_{x}^{\alpha}\left(u_{2} v\right)-2 D_{x}^{\alpha}\left(w u_{1}\right) .
\end{gathered}
$$

\section{Reduction Cases}

Case 1. When $\alpha=\beta=1, u_{1}=u_{2}=0, t_{2}=t$; (52) reduces to the BK hierarchy

$$
\begin{gathered}
v_{t}=-\frac{1}{2} v_{x x}+v v_{x}+w_{x}, \\
w_{t}=\left(v w+\frac{1}{2} w_{x}\right)_{x} .
\end{gathered}
$$

Case 2. Let $v=-q, w=r+1+(1 / 2) v_{x}$, (53) is transformed to the classical Boussinesq equation

$$
\begin{gathered}
q_{t}=-q q_{x}-r_{x}, \\
r_{t}=-\frac{1}{4} q_{x x x}-(q(r+1))_{x} .
\end{gathered}
$$

\section{Conclusion}

A way to construct the Hamiltonian structure of integrable coupling of fractional soliton equation hierarchy is presented. As an application, the Hamiltonian structure of an integrable coupling of the fractional BK hierarchy is obtained by use of the fractional quadratic-form identity. The method can be generalized to other fractional integrable couplings.

\section{Acknowledgments}

The Project is in part supported by the Natural Science Foundation of China under Grants nos. 11271008, 61072147, and 11071159, by a Grant of "The First-class Discipline of Universities in Shanghai" and the Shanghai Universty Leading Academic Discipline Project (A.13-0101-12-004), the Natural Science Foundation of Shandong Province (Grant Nos. ZR2012AM021, and ZR2012AQ011), and Scientific Research Reward Fund for Excellent Middle-Aged and Young Scientists in Shandong Province (Grants no. BS2011DX038).

\section{References}

[1] G. M. Zaslavsky, "Chaos, fractional kinetics, and anomalous transport," Physics Reports, vol. 371, no. 6, pp. 461-580, 2002.

[2] G. M. Zaslavsky, Hamiltonian Chaos and Fractional Dynamics, Oxford University Press, Oxford, UK, 2008, Reprint of the 2005 original.
[3] V. E. Tarasov and G. M. Zaslavsky, "Fractional ginzburg-landau equation for fractal media," Physica A, vol. 354, pp. 249-261, 2005.

[4] F. Riewe, "Mechanics with fractional derivatives," Physical Review E, vol. 55, no. 3, pp. 3581-3592, 1997.

[5] V. E. Tarasov, "Fractional systems and fractional Bogoliubov hierarchy equations," Physical Review E, vol. 71, no. 1, p. 12, 2005.

[6] V. E. Tarasov, "Fractional fokker-planck equation for fractal media," Chaos, vol. 15, no. 2, p. 8, 2005.

[7] V. E. Tarasov, "Fractional Liouville and BBGKI equations," Journal of Physics, vol. 7, p. 17, 2005.

[8] R. R. Nigmatullin, "Realization of the generalized transfer equation in a medium with fractal geometry," Physica Status Solidi B, vol. 133, no. 1, pp. 425-430, 1986.

[9] G. C. Wu, "A fractional lie group method for anomalous diffusion equations," Communications in Fractional Calculus, vol. 1, pp. 27-31, 2010.

[10] R. Hilfer, Applications of Fractional Calculus in Physics, World Scientific, Singarpore, 2000.

[11] R. Metzler and J. Klafter, "The restaurant at the end of the random walk: recent developments in the description of anomalous transport by fractional dynamics," Journal of Physics A, vol. 37, no. 31, pp. R161-R208, 2004.

[12] J. C. Liu and G. L. Hou, "New approximate solution for timefractional coupled $\mathrm{KdV}$ equations by generalised differential transform method," Chinese Physics B, vol. 19, no. 11, Article ID 110203, 2010.

[13] S. B. Zhou, X. R. Lin, and H. Li, "Chaotic synchronization of a fractional-order system based on washout filter control," Communications in Nonlinear Science and Numerical Simulation, vol. 16, no. 3, pp. 1533-1540, 2011.

[14] D. L. Qi, Q. Wang, and J. Yang, "Comparison between two different sliding mode controllers for a fractional-order unified chaotic system," Chinese Physics B, vol. 20, no. 10, Article ID 100505, 2011.

[15] H. X. Ge, Y. Q. Liu, and R. J. Cheng, "Element-free Galerkin (EFG) method for analysis of the time-fractional partial differential equations," Chinese Physics B, vol. 21, no. 1, Article ID 010206, 2012

[16] K. B. Oldham and J. Spanier, The Fractional Calculus, Academic Press, New York, NY, USA, 1974.

[17] S. G. Samko, A. A. Kilbas, and O. I. Marichev, Fractional Integrals and Derivatives, Gordon and Breach Science Publishers, New York, NY, USA, 1993.

[18] D. Baleanu, Z. B. Guvenc, and J. A. Machado, Tenreiro, Springer, 2009.

[19] I. Podlubny, Fractional Differential Equations, Academic, New York, NY, USA, 1999.

[20] G. M. Zaslavsky, Ed., Hamiltonian Chaos and Fractional Dynamics, Oxford University Press, New York, NY, USA, 2005.

[21] F. Riewe, "Nonconservative Lagrangian and Hamiltonian mechanics," Physical Review E, vol. 53, no. 2, pp. 1890-1899, 1996.

[22] G. S. F. Frederico and D. F. M. Torres, "Fractional conservation laws in optimal control theory," Nonlinear Dynamics, vol. 53, no. 3, pp. 215-222, 2008.

[23] D. Baleanu and S. I. Muslih, "Lagrangian formulation of classical fields within Riemann-Liouville fractional derivatives," Physica Scripta, vol. 72, no. 2-3, pp. 119-121, 2005. 
[24] D. Baleanu and Om. P. Agrawal, "Fractional Hamilton formalism within Caputo's derivative," Czechoslovak Journal of Physics, vol. 56, no. 10-11, pp. 1087-1092, 2006.

[25] R. A. El-Nabulsi, "Modifications at large distances from fractional and fractal arguments," Fractals, vol. 18, no. 2, pp. 185-190, 2010.

[26] R. A. El-Nabulsi, "A fractional action-like variational approach of some classical, quantum and geometrical dynamics," International Journal of Applied Mathematics, vol. 17, no. 3, pp. 299-317, 2005.

[27] R. A. El-Nabulsi and D. F. M. Torres, "Fractional actionlike variational problems," Journal of Mathematical Physics, vol. 49, no. 5, p. $053521,2008$.

[28] O. P. Agrawal, "Fractional variational calculus and the transversality conditions," Journal of Physics A, vol. 39, no. 33, pp. 1037510384, 2006.

[29] O. P. Agrawal, "Fractional variational calculus in terms of Riesz fractional derivatives," Journal of Physics A, vol. 40, no. 24, pp. 6287-6303, 2007.

[30] V. E. Tarasov, "Fractional variations for dynamical systems: hamilton and Lagrange approaches," Journal of Physics A, vol. 39, no. 26, pp. 8409-8425, 2006.

[31] G. Jumarie, "Lagrangian mechanics of fractional order, Hamilton-Jacobi fractional PDE and Taylor's series of nondifferentiable functions," Chaos, Solitons and Fractals, vol. 32, no. 3, pp. 969-987, 2007.

[32] R. Almeida, A. B. Malinowska, and D. F. M. Torres, "A fractional calculus of variations for multiple integrals with application to vibrating string," Journal of Mathematical Physics, vol. 51, no. 3, p. 12, 2010.

[33] A. B. Malinowska, M. R. S. Ammi, and D. F. M. Torres, "Composition functional in fractional calculus of variations," Communications in Fractional Calculus, vol. 1, p. 32, 2010.

[34] G. Z. Tu, "The trace identity, a powerful tool for constructing the Hamiltonian structure of integrable systems," Journal of Mathematical Physics, vol. 30, no. 2, pp. 330-338, 1989.

[35] W. X. Ma, "A new family of Liouville integrable generalized Hamilton equations and its reduction," Chinese Annals of Mathematics A, vol. 13, no. 1, pp. 115-123, 1992.

[36] Y. Zhang and H. Zhang, "A direct method for integrable couplings of TD hierarchy," Journal of Mathematical Physics, vol. 43, no. 1, pp. 466-472, 2002.

[37] T. Xia, F. You, and D. Chen, "A generalized AKNS hierarchy and its bi-Hamiltonian structures," Chaos, Solitons \& Fractals, vol. 23, no. 5, pp. 1911-1919, 2005.

[38] W. X. Ma and M. Chen, "Hamiltonian and quasi-Hamiltonian structures associated with semi-direct sums of Lie algebras," Journal of Physics A, vol. 39, no. 34, Article ID 10787, 2006.

[39] W.-X. Ma, X.-X. Xu, and Y. Zhang, "Semidirect sums of Lie algebras and discrete integrable couplings," Journal of Mathematical Physics, vol. 47, no. 5, p. 16, 2006.

[40] G.-c. Wu and S. Zhang, "A generalized Tu formula and Hamiltonian structures of fractional AKNS hierarchy," Physics Letters A, vol. 375, no. 42, pp. 3659-3663, 2011.

[41] F. Yu, "A generalized fractional $\mathrm{KN}$ equation hierarchy and its fractional Hamiltonian structure," Computers \& Mathematics with Applications, vol. 62, no. 3, pp. 1522-1530, 2011.

[42] H. Wang and T. C. Xia, "The fractional supertrace identity and its application to the super Ablowitz-Kaup-Newell-Segur hierarchy," Journal of Mathematical Physics, vol. 54, no. 5, Article ID 043505, 2013.
[43] H. Wang and T.-C. Xia, "The fractional supertrace identity and its application to the super Jaulent-Miodek hierarchy," Communications in Nonlinear Science and Numerical Simulation, vol. 18, no. 10, pp. 2859-2867, 2013.

[44] K. M. Kolwankar and A. D. Gangal, "Fractional differentiability of nowhere differentiable functions and dimensions," Chaos, vol. 6, no. 4, pp. 505-513, 1996.

[45] K. M. Kolwankar and A. D. Gangal, "Hölder exponents of irregular signals and local fractional derivatives," Pramana, vol. 48, no. 1, pp. 49-68, 1997.

[46] K. M. Kolwankar and A. D. Gangal, "Local fractional FokkerPlanck equation,” Physical Review Letters, vol. 80, no. 2, pp. 214217, 1998.

[47] W. Chen, "Time-space fabric underlying anomalous diffusion," Chaos, Solitons \& Fractals, vol. 28, no. 4, pp. 923-929, 2006.

[48] W. Chen and H. G. Sun, "Multiscale statistical model of fullydeveloped turbulence particle accelerations," Modern Physics Letters B, vol. 23, no. 3, p. 449, 2009.

[49] J. Cresson, "Scale calculus and the Schrödinger equation," Journal of Mathematical Physics, vol. 44, no. 11, pp. 4907-4938, 2003.

[50] J. Cresson, "Non-differentiable variational principles," Journal of Mathematical Analysis and Applications, vol. 307, no. 1, pp. 48-64, 2005.

[51] A. Parvate and A. D. Gangal, "Calculus on fractal subsets of real line-I. Formulation," Fractals, vol. 17, no. 1, pp. 53-81, 2009.

[52] G. Jumarie, "Modified Riemann-Liouville derivative and fractional Taylor series of nondifferentiable functions further results," Computers \& Mathematics with Applications, vol. 51, no. 9-10, pp. 1367-1376, 2006.

[53] G. Jumarie, "Table of some basic fractional calculus formulae derived from a modified Riemann-Liouville derivative for nondifferentiable functions," Applied Mathematics Letters, vol. 22, no. 3, pp. 378-385, 2009.

[54] Y. Chen, Y. Yan, and K. Zhang, "On the local fractional derivative," Journal of Mathematical Analysis and Applications, vol. 362, no. 1, pp. 17-33, 2010.

[55] X. J. Yang, Research on fractal mathematics and some applications in mechanics [M.S. thesis], China University of Mining and Technology, 2009, Chinese.

[56] F. Ben Adda, "Geometric interpretation of the fractional derivative," Journal of Fractional Calculus, vol. 11, pp. 21-51, 1997.

[57] F. Ben Adda and J. Cresson, "About non-differentiable functions," Journal of Mathematical Analysis and Applications, vol. 263, no. 2, pp. 721-737, 2001.

[58] K. Cottrill-Shepherd and M. Naber, "Fractional differential forms," Journal of Mathematical Physics, vol. 42, no. 5, pp. 22032212, 2001.

[59] K. K. Kazbekov, "Fractional differential forms in euclidean space," Vladikavkazskiı̆ Matematicheskiı Zhurnal, vol. 7, no. 2, pp. 41-54, 2005.

[60] C. Li and W. Deng, "Remarks on fractional derivatives," Applied Mathematics and Computation, vol. 187, no. 2, pp. 777-784, 2007.

[61] F. Guo and Y. Zhang, "The quadratic-form identity for constructing the Hamiltonian structure of integrable systems," Journal of Physics A, vol. 38, no. 40, pp. 8537-8548, 2005. 


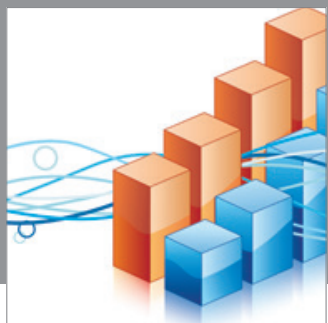

Advances in

Operations Research

mansans

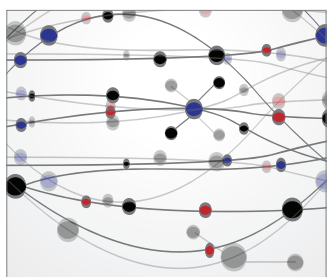

The Scientific World Journal
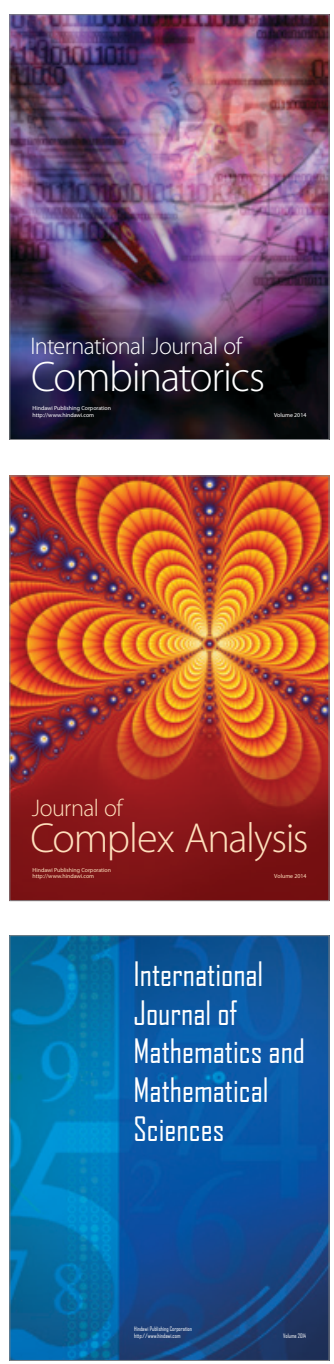
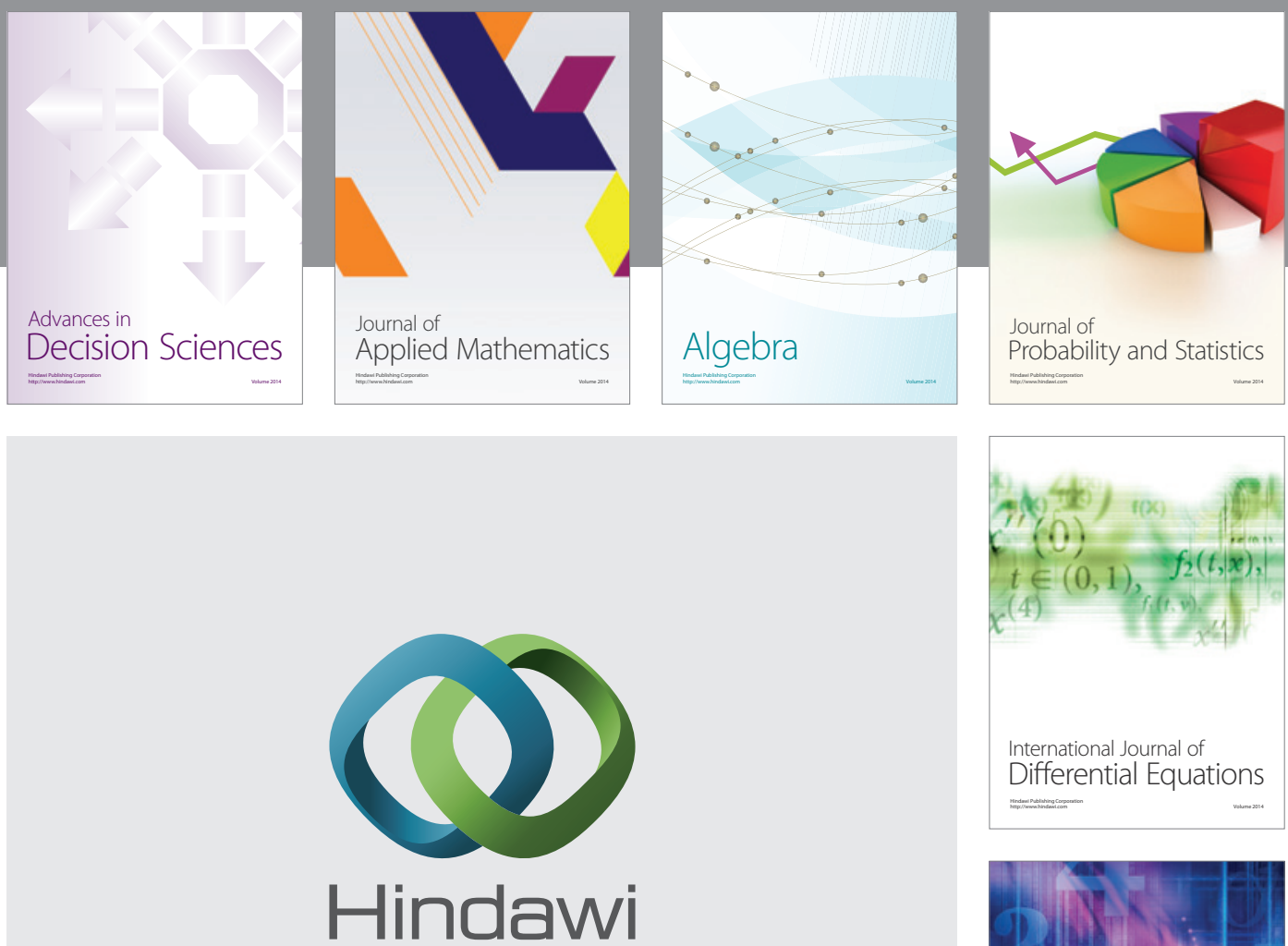

Submit your manuscripts at http://www.hindawi.com
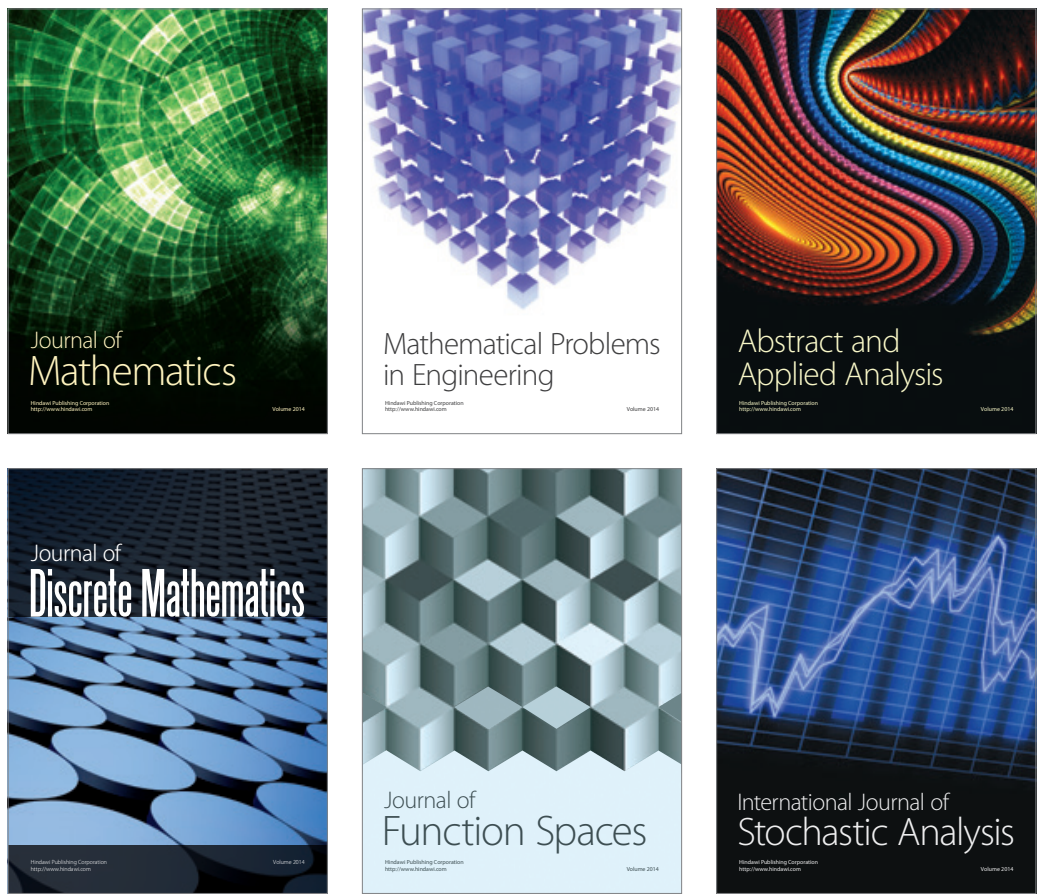

Journal of

Function Spaces

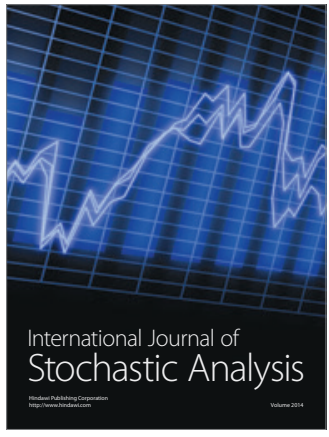

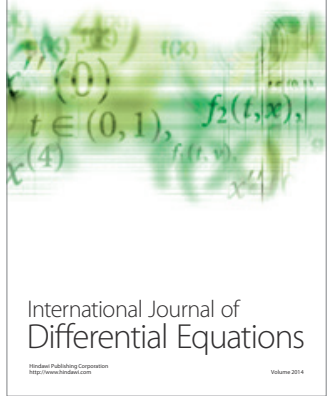
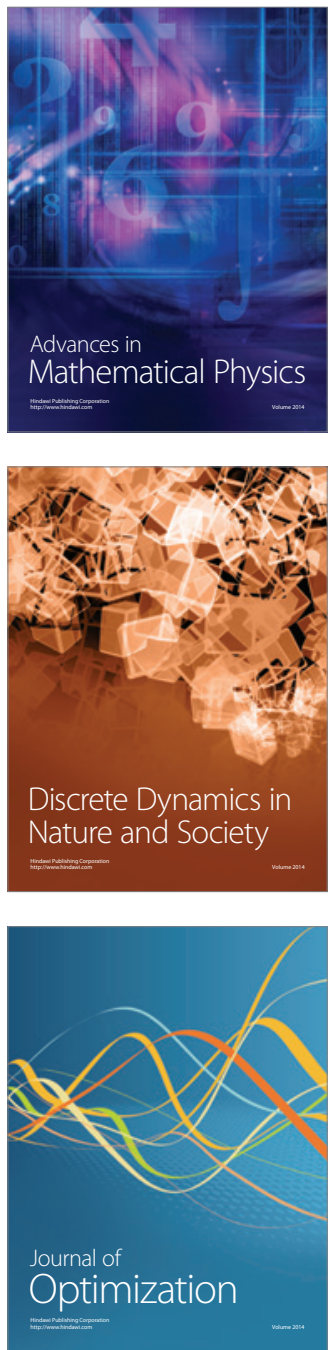